\title{
INDICE DELL'ANNATA 1996
}

\section{SAGGI}

Riflessioni sulla metodologia degli studi comparati di Neil J. Smelser

Integrazione europea e welfare states nazionali

di Fritz W. Sharpf

Cosa è «competizione» in politica e come va studiata

L'espansione del potere giudiziario nelle democrazie contemporanee

di Carlo Guarnieri e Patrizia Pederzoli

\section{RICERCHE}

Il modello sud-europeo di welfare state di Maurizio Ferrera

Dal pluralismo polarizzato al pluralismo moderato. Il modello di Sartori e la transizione italiana di Adriano Pappalardo

La formazione dei partiti confessionali in Europa di Stathis N. Kalyvas

\section{NOTE}

Federalismo e movimenti sociali di Marco G. Giugni 
Alla ricerca della collegialità di governo: i vertici di maggioranza dal 1970 al 1994

di Annarita Criscitiello

I gruppi parlamentari dopo il 1994. Fluidità e riaggregazioni di Luca Verzichelli

\section{ELEZIONI POLITICHE 1996}

Programmi, media e opinione pubblica

di Giacomo Sani e Paolo Segatti

I candidati. Visioni politiche e carriere

di Liborio Mattina e Alessandro Tonarelli

Le alleanze elettorali. Identità partitiche e logiche coalizionali

di Aldo Di Virgilio

La partecipazione elettorale: gli effetti della competizione maggioritaria

di Daniele Caramani

Indizi di un inverno precoce: il voto proporzionale tra equilibrio e continuità di Roberto Cartocci

Come perdere una maggioranza. La competizione nei collegi uninominali di Roberto D'Alimonte e Stefano Bartolini

L'effetto mancato della riforma maggioritaria: il voto strategico di Alessandro Chiaramonte

La classe politica della transizione di Luca Verzichelli

\section{RECENSIONI}

A. Agh (a cura di), The Emergence of East Central European Parliaments: the First Steps [Maurizio Cotta] 
A. Agh e S. Kurtan (a cura di), Democratization and Europeanization in Hungary: the First Parliament (1990 1994) [Maurizio Cotta]

A. Agh e G. Ilonski (a cura di), Parliament and Organized Interests: the Second Steps [Maurizio Cotta]

S.S. Andersen e K.A. Eliassen (a cura di), Making Policy in Europe. The Europeification of national Policy-Making [Marco Giuliani]

F. Attinà, Il sistema politico della Comunità Europea [Marco Giuliani]

D.S. Bell (a cura di), Western European Communists and the Collapse of Communism [Anna Bosco]

V. Belotti e M. Maraffi, Ceto politico e dirigenza amministrativa nei comuni italiani [Gian Luigi Bulsei]

M.J. Bull e P. Heywood (a cura di), West European Communist Parties after the Revolutions of 1989 [Anna Bosco]

D. Butler, A. Adonis e T. Travers, Failure in British Government. The Politics of the Pool Tax [Claudio M. Radaelli]

D.J. Coyle e R.J. Ellis (a cura di), Politics, Policy and Culture [Gilberto Capano]

G. Edwards e D. Spence (a cura di), The European Commission [Marco Giuliani]

J. Elster, Il cemento della società. Uno studio sull'ordine sociale [Nicola Pasini]

S. Fabbrini, Il presidenzialismo americano [Luciano Cavalli]

M. Fedele e R. Leonardi (a cura di), La politica senza $i$ partiti [Luca Verzichelli]

D. Fisichella, Epistemologia e scienza politica [Alberto Marradi] 
S. Haggard e R. Kaufman, The political Economy of Democratic Transition [Fabio Fossati]

R.S. Katz e P. Mair (a cura di), Party Organizations: a Data Handbook on Party Organization in Western Democracies, 1960-90 [Piero Ignazi]

R.S. Katz e P. Mair (a cura di), How Parties Organize. Change and Adaptation in Party Organizations in Western Democracies [Piero Ignazi]

R. Kent Weaver e B.A. Rockman (a cura di), Do Institutions matter? Government Capabilities in the United States and Abroad [Marco Giuliani]

H.D. Klingemann, R.I. Hofferbert e I. Budge, Parties, Policies and Democracy [Salvatore Vassallo]

P. Ignazi, L'estrema destra in Europa [Luca Verzichelli]

B. Jobert (a cura di), Le tournant néo-libéral en Europe [Gilberto Capano]

M. Laver e K.A. Shepsle (a cura di), Cabinet Ministers and Parliamentary Government [Daniela Giannetti]

J.J. Linz e A. Valenzuela (a cura di), Il fallimento del presidenzialismo [Sergio Fabbrini]

G. Nevola, Conflitto e coercizione. Modello di analisi e studio di casi [Gigi Graziano]

B.G. Peters e A. Barker (a cura di), Advising West European Governments. Inquiries, Expertise and Public Policy [Claudio M. Radaelli]

M.S. Piretti, Le elezioni politiche in Italia dal 1848 a oggi [Daniele Caramani]

W.H. Riker (a cura di), Agenda Formation [Daniela Giannetti]

W. Santagata, Economia, elezioni, interessi [Francesco Zucchini] 
M.S. Shugart e J.M. Carey, Presidenti e assemblee. Disegno costituzionale e dinamiche elettorali [Sergio Fabbrini]

S. Vassallo, Il governo di partito in Italia (1943-1993) [Francesco Zucchini]

L. Weinberg, The Transformation of Italian Communism [Anna Bosco] 